\section{P799 ACCELERATED HIV CASE FINDING AND BRIDGING ENROLMENT GAP FOR KEY POPULATIONS IN WESTERN NIGERIA: A BREAK-EVEN IN THE 90-90-90}

Saheed Usman*, Femi Owolagba, Adetosoye Adebanjo, Jennifer Ale, Chisom Udechukwu, Babatunde Akinbinu, Chiedozi Akueshi, Funmilola Elusoji, Michael Tomori, Ererosanaga Ogboghodo. APIN Public Health Intiatives, Abuja, Nigeria

\subsection{6/sextrans-2019-sti.852}

Background The clock is steadily ticking towards 2020 when the UNAIDS 90-90-90 global target in the fight against HIV/ AIDS is hoped to be achieved. The hypothesis tested is the significant association between youthful age and HIV test outcome. The aim of the study was to engage in an accelerated HIV case finding and ensure enrolment into care among key populations in Western Nigeria fulfilling the first \& second 90 of the UNAIDS targets.

Methods Lay Adhoc Staff/volunteers were purposely selected and trained. Consenting prison inmates had their blood samples taken and tested following the country's HIV serology National testing algorithm, using the recommended HIV testing kits. Those who tested positive went through a retesting process in the laboratory and confirmed positive. Post-test counselling was then conducted.

Results A total of 771 prison inmates were tested across the four prisons (Male 765, Female 6) with a mean age \pm SD is $31.25 \pm 9.47$ years. Ten of them (Male 9, Female 1) were confirmed new positives with a mean age \pm SD is $31.40 \pm$ 6.24 years, yielding a positivity rate of $1.3 \%$. Eight of the ten positives are in their youthful age ( $<35$ years). Odd's ratio shows that youthful age have higher association with HIV test outcome (OR: 2.81, CI: 0.80-9.79). The linkage rate for the positives is $100 \%$ with good escort service while adherence is $\geq 95 \%$.

Conclusion This mode of HIV testing service (HTS) has proved to reach a key population yielding more positives in much fewer numbers of people tested and in a short period of time with $100 \%$ linkage with better resource/health financing outlook. Community ART Differentiated Service Delivery (DSD) Model is in line for the patients to sustain the gains in the effort to achieve the 90-90-90 fast track UNAIDS targets. Disclosure No significant relationships.

\section{\begin{tabular}{|l|l}
\hline P800 & PREVALENCE OF CURABLE SEXUALLY TRANSMITTED
\end{tabular} INFECTIONS AMONG REFUGEES: GLOBAL SYSTEMATIC REVIEW AND META-ANALYSIS}

${ }^{1}$ Ghina Mumtaz*, ${ }^{1}$ Eman Sharara, ${ }^{1} J o c e l y n$ Dejong, ${ }^{1}$ Abla Sibai, ${ }^{2}$ Bayard Roberts, ${ }^{3}$ Laith Abu-Raddad. 'American University of Beirut, Epidemiology and Population Health, Beirut, Lebanon; ${ }^{2}$ London School of Hygiene and Tropical Medicine, London, UK; ${ }^{3}$ Weill Cornell Medicine-Qatar, Doha, Qatar

10.1136/sextrans-2019-sti.853

Background The world is witnessing one of the worst refugee crises of all times. Disrupted healthcare systems, limited availability and access to services, poverty, and increased exposure to sexual violence among others increase the vulnerability of populations in humanitarian settings to poor sexual/reproductive outcomes, including sexually transmitted infections (STIs). Our objective is to characterize, for the first time, the epidemiology of curable STIs - Chlamydia trachomatis (CT),
Neisseria gonorrhoeae (NG), Treponema pallidum (syphilis), and Trichomonas vaginalis (TV) - among refugees and internally displaced populations globally, and to estimate their pooled mean prevalence.

Methods We conducted a PRISMA-guided systematic review of literature through PubMed and Embase databases, and of abstracts of international HIV/STI conferences, with no language/time restrictions. Pooled prevalence of current and/or lifetime infection for each STI was estimated using randomeffects meta-analyses.

Results We identified 37 eligible studies that contributed 103 STI prevalence measures for 935,191 refugees. The majority of studies were for syphilis (65\%), whereas CT, NG, and TV accounted for $14 \%, 18 \%$, and $4 \%$ of total measures, respectively. African and South-east Asian refugees were represented the most. Only one study was conducted among Syrian refugees - currently the largest refugee population worldwide. Pooled prevalence of current infection was 1.6\% (95\% CI: 1.2-2.1\%) for syphilis, $0.6 \%$ (95\% CI: $0.1-1.4 \%)$ for $\mathrm{NG}$, $0.5 \%$ (95\% CI: $0.2-1.0 \%)$ for CT, and $29.3 \%$ (95\% CI: 21.3-38.0\%) for TV. The latter included mostly symptomatic populations. Pooled prevalence of lifetime syphilis infection was $3.3 \%$ (95I CI: $1.5-5.8 \%$ ).

Conclusion Despite the identified studies, there is a large knowledge gap for these infections in this population. Findings suggest overall comparable prevalence levels to the general population. However, further studies are needed to better understand the recent burden of STIs and the sexual health needs of affected populations in different global settings, to inform screening and treatment policy and programming.

Disclosure No significant relationships.

\section{P802 SIZE OF FEMALE INJECTING DRUG USERS IN 10 NIGERIAN STATES AND IMPLICATIONS FOR HIV AND REPRODUCTIVE HEALTH}

Idoteyin Ezirim*, Olutosin Adebanjo. National Agency for the Control of AIDS, Research, Monitoring and Evaluation, Abuja, Nigeria

\subsection{6/sextrans-2019-sti.854}

Background HIV prevalence is high amongst key population (KPs). People who inject drugs (PWID) are considered as KPs because certain behaviors increase their vulnerability to HIV. To reach PWIDS with interventions, there's need to determine their distribution and size. Previously, gender wasn't considered in PWID size estimation, therefore designing HIV/Reproductive health services for female PWIDS was difficult because their size was unknown. To address this, Nigeria conducted programmatic mapping to provide insight on size of male and female PWIDS and geographical locations.

Methods PWIDs were mapped in all Local Government Areas in10 states. We employed programmatic mapping approach which involved two sequential data collection steps called level one [L1] and level two [L2]. During L1, information was collected from key informants (KIs) about the geographic locations/spots where PWIDS congregate, the characteristics of the spots (public place, bunk, etc.) and an estimate of number of PWIDs who can be found there. L1 interviews were conducted at markets, parks, streets, etc. During L2, KI interviews were conducted at spots identified in L1. In L2 interviews, 